\title{
Volume doubling time of lung adenocarcinomas considering epidermal growth factor receptor mutation status of exon 19 and 21: three-dimensional volumetric evaluation
}

\author{
Rui Zhang ${ }^{1}$, Bojiang Chen ${ }^{1}$, Yongzhao Zhou ${ }^{1}$, Ping Zhou ${ }^{2}$, Jing Jin ${ }^{1}$, Longchao Zhao ${ }^{3}$, Weimin Li $^{1}$ \\ ${ }^{1}$ Department of Respiratory and Critical Care, ${ }^{2}$ Department of Pathology, ${ }^{3}$ Department of Health Related Social and Behavioral Science, West China \\ Hospital, Sichuan University, Chengdu 610041, China \\ Contributions: (I) Conception and design: W Li, R Zhang; (II) Administrative support: W Li; (III) Provision of study materials or patients: B Chen \\ and P Zhou; (IV) Collection and assembly of data: R Zhang, Y Zhou; (V) Data analysis and interpretation: J Jin, L Zhao; (VI) Manuscript writing: All \\ authors; (VII) Final approval of manuscript: All authors. \\ Correspondence to: Weimin Li. Department of Respiratory and Critical Care Medicine, West China Hospital, No. 37 Guo Xue Alley, Chengdu \\ 610041, China. Email: weimi003@yahoo.com.
}

Background: The current study was to investigate the volume doubling time (VDT) of lung adenocarcinomas considering epidermal growth factor receptor (EGFR) mutation status of exon 19 and 21 , when compared with EGFR wide type.

Methods: Eighty-eight patients with pathologically proven adenocarcinomas, which underwent two or more computed tomography (CT) scans spared by 25 or more days, were included. EGFR mutations at exons 19 and 21 were determined using amplification refractory mutation system and all patients were divided into three groups-EGFR wide type group, EGFR mutation in exon 19 and 21 groups. Threedimensional manual segmentations for all tumors were performed on first and latest follow-up CT scans; subsequently, VDTs were calculated and compared among three groups. Clinicopathoradiologic characters were also collected for subgroup analysis.

Results: EGFR mutations occurred in 49 (55.7\%) patients, 19 in exon 19 and 30 in exon 21, respectively. The median VDT of all patients (33 men, 55 women; median age, 62 years) was 214 days (range, -4,092 to 10,920 days). Highly differentiated adenocarcinomas (median, 408 days) demonstrated longer VDT than those moderately (median, 172 days) or poorly (median, 144 days) differentiated $(\mathrm{P}=0.04)$. The VDT distribution was similar among EGFR wide type group (median, 207 days), EGFR mutation in exon 19 group (median, 288 days) and exon 21 group (median, 144 days) $(\mathrm{P}=0.21)$. In subgroup analysis, the median VDT of adenocarcinomas with EGFR mutation in exon 19 was longer than that of EGFR wide type for males $(\mathrm{P}=0.03)$ or patients without spiculation sign in chest CT $(\mathrm{P}=0.04)$. Totally 24 adenocarcinomas presented negative VDTs, most of which tended to be stable. Positive VDT values were used for all median description.

Conclusions: Overall VDT of lung adenocarcinomas seems not affected by EGFR mutation status. Researches with large population are warranted for further study.

Keywords: Adenocarcinoma; volume doubling time (VDT); epidermal growth factor receptor (EGFR) mutation; exon 19; exon 21

Submitted May 10, 2017. Accepted for publication Oct 10, 2017.

doi: $10.21037 /$ jtd.2017.10.58

View this article at: http://dx.doi.org/10.21037/jtd.2017.10.58 


\section{Introduction}

Epidermal growth factor receptor (EGFR), a member of the ErbB receptor tyrosine kinase family, harbors tumor associated mutations that have been documented in exon 18 through 21, mainly involving exon 19 and exon 21. Previous studies have revealed that EGFR mutation status is closely related to the manifestation and prognosis of lung adenocarcinomas (1-3). In advanced non-small cell lung cancer (NSCLC) patients, the mutation in exon 19 could provide superior objective response rate (ORR), one-year progression-free survival (PFS), and two-year overall survival (OS) after EGFR-tyrosine kinase inhibitors (EGFR-TKIs) treatment when compared with mutation in exon 21 (4-6). Nevertheless, patients with mutation in exon 19 may have few choices available during gradual progression and sometimes obtain survival benefit from chemotherapy during dramatic progression, while patients with dramatic or gradual progression could both benefit from previous TKI continuation in exon 21 mutation patients (7). Therefore, the EGFR mutation subtypes should be considered when making treatment decision for EGFR mutation-positive NSCLC patients.

However, detection of EGFR mutation can fail for lots of reasons, such as inoperability, sampling artifact, small biopsy specimens, tumor heterogeneity or low sensitivity of mutation detection methods. Hence, there should be some indications of EGFR mutation status for physicians to determine what treatment is appropriate at the right moment and if the repeated EGFR mutation test should be performed. Volume doubling time (VDT), the time taken for a tumor to double in volume, has been used to determine dynamic characterization of tumor growth $(8,9)$. Based on three-dimensional measurements, it can provide valuable information of whether a lung cancer is growing or stable $(10,11)$. If the patient is undergoing active surveillance, such message means a lot for precise and intermediate intervention. Besides, it could indicate some natural features of the tumor, such as diver gene mutation status. Nakamura $\mathrm{et} \mathrm{al}$. firstly described the possible relationships between VDT and EGFR mutation status, finding positive EGFR mutation status may be associated with longer VDT. However, the major limitation of Nakamura's study is the VDT calculation depended on conventional measurement methods and possible statistical bias could have been caused by squamous-cell carcinoma (12).

The current study has overcome the limitations mentioned above and intended to throw more light on growth variability among EGFR wide type group, EGFR mutation in exon 19 and exon 21 groups. Besides, clinicopathoradiologic characters were also collected for subgroup analysis.

\section{Methods}

\section{Patients}

This retrospective study had institutional review board approval. From January 2014 to July 2016, initially 1,613 patients were selected with diagnosis of pulmonary adenocarcinoma in West China Hospital. Medical records were carefully reviewed of selected cases for study eligibility. Patients were included if the following criteria were met: (I) available pathology reports with diagnosis of lung adenocarcinoma; (II) available test results of EGFR mutation status; (III) at least two computed tomography (CT) scans available in our picture achieving and communication system (PACS) prior to any clinical treatments, such as surgery, radiotherapy or chemotherapy, and two CT scans spaced a minimum of 25 days apart (13). Patients were excluded because of the following excluded criteria: (I) incomplete images; (II) extraction failure of images from PACS to threedimension reconstruction software, (III) difficulties in lesion segmentation due to large pleural effusion or secondary obstructive pneumonia or pleural attachment. When there were multiple nodules, the largest one was selected. In total, 88 nodules in 88 patients were studied. Clinical Data were collected for analysis, including age, sex, smoking history, pathologic results, surgery records, clinical stage and EGFR mutation status.

\section{CT image acquisition}

Among 88 patients, chest CT examinations were performed for routine clinical care by using one of three multidetector CT systems (Siemens Medical Systems, Forchheim, Germany; Brilliance 64, Philips Medical Systems, Eindhoven, the Netherlands; Somatom Definition, Siemens Healthcare, Forchheim, Germany). Scanning parameters were as followings: (I) $120 \mathrm{kV}, 100 \mathrm{mAs}$, rotation time of $0.5 \mathrm{~s}$, collimation of $16 \mathrm{~mm} \times 0.75 \mathrm{~mm}$, pitch of 0.85 ; (II) $120 \mathrm{kV}$, $145 \mathrm{mAs}$, rotation time of $0.42 \mathrm{~s}$, collimation of $64 \mathrm{~mm} \times$ $0.625 \mathrm{~mm}$, pitch of 0.891 ; (III) $120 \mathrm{kV}, 200 \mathrm{mAs}$, rotation time of $0.33 \mathrm{~s}$, collimation of $24 \mathrm{~mm} \times 1.2 \mathrm{~mm}$, pitch of 0.9. Totally 176 scans of all patients were applied for VDT calculation, 76 with cut thickness of $1 \mathrm{~mm}$ and 100 with cut thickness of $5 \mathrm{~mm}$. 


\section{$V D T$}

If there were multiple scans available for one patient, images with similar slice thickness and consistently with or without intravenous contrast were paired for analysis. When two specific CT scans were selected for each patient, one physician, who has been trained to use threedimensional reconstruction software (IQQA-Chest, EDDA Technology, Princeton Junction, NJ, USA), performed three-dimensional manual segmentation of all 88 tumors on the initial CT scans. After 1 month, the same observer performed manual segmentation of the tumors on the last CT scans. The observer was blind to results of EGFR mutation tests. Once manual segmentation was finished, a three-dimensional lesion was visible with volume, mass and size of the tumor being calculated. The VDT then can be calculated based on the following equation (14):

$\mathrm{VDT}=[\mathrm{t} \log 2] /[\log \mathrm{Vt} / \mathrm{V} 0]$

Where $t$ is time between scans, $\mathrm{Vt}$ is the second scan volume and $\mathrm{V} 0$ is the first scan volume.

\section{Image interpretation}

One clinical physician (B Chen) in Pulmonary and Critical Department, with 10 years of experience in chest CT, read the entire 88 images initially taken. Five of the following sensitive radiological characters of the studied tumor in each patient were recorded: (I) text, recorded as "solid" if the whole nodule was composed of solid component, otherwise recorded as mixed ground glass opacity (mGGO) or pure ground glass opacity (pGGO); (II) spiculation, recorded as "yes" if there were lines radiating from the margins of the tumor, otherwise recorded as "no"; (III) pleural retraction, recorded as "yes" when the pleura was retracted toward tumor, otherwise recorded as "no"; (IV) air bronchogram, recorded as "yes" if tubelike or branched air structure was presented within the tumor, otherwise recorded as "no"; (V) emphysema, recorded as "yes" if the pulmonary emphysema was more than $25 \%$, otherwise recorded as "no". Besides, another physician (Y Zhou, with 5 years of experience in chest CT) in Pulmonary and Critical Department independently read the initial images of all 88 tumors. Both physicians were blind to clinical findings of tumors. The agreement for classification of radiological features between two readers was good. Kappa value for classifications of tumor texture, spiculation sign, pleural retraction, air bronchogram, and emphysema were $0.74,0.74$, $0.68,0.75$ and 0.90 , respectively.

\section{Detection of EGFR mutation}

To examine EGFR mutation status, tumor specimens fixed in $10 \%$ formalin and embedded in paraffin were sent to the Pathology Department in our Hospital. Molecular analysis of mutation was performed with Cycleave realtime quantitative PCR technique (ADx-ARMs) by using the EGFR Gene Mutation Detection Kit (AmoyDx ${ }^{\circledR}$, Xiamen, China).

\section{Data and statistical analysis}

In this study, as the numerical data sets were not normally distributed, they were presented with median and range, while categorical data were described in number and percentage. Statistical comparisons between two groups were performed by Mann-Whitney U test. Statistical significances among EGFR wipe type group, EGFR mutation in exon 19 and 21 groups were determined by Kruskal-Wallis test. The agreement between two readers was tested with Kappa index. All analysis was performed using SPSS software 20.0. $\mathrm{P}<0.05$ was considered statistically significant.

\section{Results}

\section{Patient characteristics}

There were 33 men (37.5\%) and 55 women (62.5\%) with a median age of 62 years (range, 36-84 years). All 88 tumors were pathologically evaluated in our Pathological Department and the diagnosis included 5 adenocarcinomas in situ (AIS), 3 minimally invasive adenocarcinomas (MIS) and 80 invasive adenocarcinomas. There were 43 available records on histological subtype, 21 lepidic predominant adenocarcinomas and 22 other types, mainly acinar predominant lesions. As for histological grade, available 48 adenocarcinomas have been classified as poorly $(n=14)$, moderately $(n=21)$ and well differentiated malignant lesions $(n=13)$, respectively; $53(60.3 \%)$ patients were diagnosed at an early stage and $74(84.1 \%)$ were initially treated with surgery. EGFR mutation was observed in 49 patients $(55.7 \%)$, 19 in exon $19(38.8 \%)$ and 30 in exon $21(61.2 \%)$, respectively. For 2 patients with EGFR mutation in exon 21, 1 patient also harbored EGFR mutation in exon 18 (G719X), and another one had other two mutations in exon 20 (G719X) and exon 18 (G719X). The median interval between two CT scans priori to any clinical treatment was 
Table 1 Clinical characteristics and VDT of 88 studied patients

\begin{tabular}{|c|c|}
\hline Characteristics & Median [range] or No. (\%) \\
\hline Age (years) & $62[36-84]$ \\
\hline \multicolumn{2}{|l|}{ Sex } \\
\hline Male & $33(37.5)$ \\
\hline Female & $55(62.5)$ \\
\hline \multicolumn{2}{|l|}{ Smoking history } \\
\hline Never-smoker & $63(71.6)$ \\
\hline Smoker & $25(28.4)$ \\
\hline \multicolumn{2}{|l|}{ Pathologic results } \\
\hline AIS & $5(5.7)$ \\
\hline MIA & $3(3.4)$ \\
\hline Invasive adenocarcinoma & $80(90.9)$ \\
\hline \multicolumn{2}{|l|}{ Clinical stage } \\
\hline I-II & $53(60.3)$ \\
\hline III-IV & $35(39.8)$ \\
\hline \multicolumn{2}{|l|}{ EGFR mutation } \\
\hline Wide type & $39(44.3)$ \\
\hline Mutation in exon 19 & $19(21.6)$ \\
\hline Mutation in exon 21 & $30(34.1)$ \\
\hline \multicolumn{2}{|l|}{ Surgically resected } \\
\hline Yes & $74(84.1)$ \\
\hline No & $14(15.9)$ \\
\hline Scan interval (days) & $56[25-2,105]$ \\
\hline VDT $^{\#}$ (days) & $214[-4,092$ to 10,920$]$ \\
\hline
\end{tabular}

\#, the median for VDT didn't include negative values $(n=24)$. EGFR, epidermal growth factor receptor; AIS, adenocarcinoma in situ; MIS, minimally invasive adenocarcinoma; VDT, volume doubling time
56 days (range, 25-2,105 days), while the median VDT was 214 days (range, 55-10,920 days) for all patients except for $24(27.3 \%)$ patients with negative values (Table 1$)$.

\section{Three-dimensional volumetric measurements}

Table 2 shows three-dimensional volumetric measurements of tumors. Among EGFR wide type, EGFR mutation in exon 19 and 21 groups, the median initial average tumor size was $17.85 \mathrm{~mm}$ (range, 7.50-49.91 mm), $18.49 \mathrm{~mm}$ (range, 9.92-37.86 mm) and $20.57 \mathrm{~mm}$ (range, $8.40-71.36 \mathrm{~mm}$ ), with a $\mathrm{P}$ value of 0.47 . Besides, the median initial volume was $2,825 \mathrm{~mm}^{3}$ (range, 231-55,901 $\mathrm{mm}^{3}$ ), 2,599 $\mathrm{mm}^{3}$ (range, $580-30,024 \mathrm{~mm}^{3}$ ) and $5,061 \mathrm{~mm}^{3}$ (range, $428-108,811 \mathrm{~mm}^{3}$ ), $\mathrm{P}=0.25$. Except for negative values, median VDT were 207 days (range, 55-10,920 days), 288 days (range, 91-3,749 days) and 144 days (range, 61-4,148 days), respectively ( $\mathrm{P}=0.21$ ). Significant statistical difference in VDT was not observed among three groups. Growth curves of 20 adenocarcinomas with three or more comparable CT scans before any clinical treatment were shown in Figure 1. There were 8 wide-type adenocarcinomas, 6 exon 19 mutation adenocarcinomas and 6 exon 21 mutation adenocarcinomas. Wide-type and EGFR-mutant-positive adenocarcinomas showed almost similar growth pattern.

\section{Clinicopathoradiologic characters and VDT}

Table 3 shows how VDT distributed at different clinicopathoradiologic levels for three groups. Firstly, when comparing VDT at different clinicoradiological levels, we found significant differences were observed among three groups for males $(\mathrm{P}=0.03)$ and patients who didn't harbor spiculation sign in chest CT $(\mathrm{P}=0.04)$. At these two levels, VDT distribution of exon19 group was different from

Table 2 Three-dimensional volumetric measurements of 88 tumors

\begin{tabular}{|c|c|c|c|c|}
\hline Characteristics & Wide type & Mutation in exon 19 & Mutation in exon 21 & $P$ value \\
\hline Initial mass (g) & $0.59(0.05-53.26)$ & $0.83(0.1-9.08)$ & $1.26(0.05-81.01)$ & 0.43 \\
\hline Initial attenuation value $(\mathrm{HU})$ & $-345(-758$ to 60$)$ & $-396(-645$ to 37$)$ & $-367(-758$ to 67$)$ & 0.83 \\
\hline Initial volume $\left[\mathrm{mm}^{3}\right]$ & $2,825[231-55,901]$ & $2,599[580-30,024]$ & $5,061[428-108,811]$ & 0.25 \\
\hline VDT $^{\#}$ (days) & $207(-4,092$ to 10,920$)$ & $288(-3,134$ to 3,749$)$ & $144(-855$ to 4,148$)$ & 0.21 \\
\hline
\end{tabular}

", the median for VDT didn't include negative values [n=24); * calculated with the Kruskal-Wallis test. VDT, volume doubling time, HU, Hounsfield unit. 
that of EGFR wide type group. Adenocarcinomas with exon 19 deletion always had a slowly growing tendency, with a median VDT being 273 and 433 days, respectively. However, wide-type adenocarcinomas presented larger growth variation, either growing much quicker (median VDT, 164 and 318 days, respectively) or staying stable (more negative values) (Figure 2). The receiver operating characteristic (ROC) curves were conducted using VDT for males (AUC, 0.784; 95\% CI, 0.608-0.960) and patients without spiculation sign (AUC, 0.778; 95\% CI, 0.614-0.942). Nevertheless, the specificities weren't high enough and the cut-off value was 139 and 99 days, respectively. In other levels, the VDT of three groups did vary, though statistical differences weren't found.

Secondly, when came to pathological analyses, highly differentiated adenocarcinomas tended to grow slowly, and the median VDT for poorly, moderately and well differentiated adenocarcinomas were 144, 172 and 408 days, respectively $(\mathrm{P}=0.04)$. The median VDT for lepidic predominant adenocarcinomas and other types were 266 and 303 days, respectively $(\mathrm{P}=0.50)$. For the patients in stage I/II and stage III/IV, the tumors seemed to double at the similar speed, the time being 220 and 207 days, respectively $(\mathrm{P}=0.82)$. However, the strong associations between EGFR mutation status and VDT weren't observed within each subgroup. All positive values were used for median description.

\section{Negative VDT}

Totally, 24 patients presented negative VDT, 8 males and 16 females with a median age of 59 years (range, 36-80 years). Median volume decreased was $-442 \mathrm{~mm}^{3}$ (range, -15 to $12,795 \mathrm{~mm}^{3}$ ) and the median scan interval was 58 days (range, 25-431 days). The relative volume decreased ranged from $1 \%$ to $53 \%$. One patient without EGFR mutation had the largest calculated shrinkage, from 786 to $373 \mathrm{~mm}^{3}$, while the visualized tumor shrinkage was not that obvious. The negative values were found 14 in wide type group (range, 1-53\%), 3 in EGFR mutation in exon 19 group (range, 2-6\%) and 7 in exon 21 group (range, 10-46\%) (Figure 3).

\section{Discussion}

In our studied patients with adenocarcinoma, $62.5 \%$ were female and therefore $71.6 \%$ were non-smokers. Except for 24 negative values, the median VDT of the other 64 adenocarcinomas was 214 days, ranging from 55 to 10,920 days; this finding was consistent with previous studies (15-17). Highly differentiated adenocarcinomas were less aggressive than those moderately or poorly differentiated, with a median VDT being 408, 172 and 144 days, respectively. The median VDT of EGFR wide type group, EGFR mutation in exon 19 and exon 21 groups was 207, 288 and 144 days, respectively. Significant difference was not observed among three groups. There were $40(62.5 \%)$ fast growing tumors, with a VDT less than 400 days, while 18 (28.1\%) adenocarcinomas grew slowly and the VDT was more than 600 days. Among the slow-growing adenocarcinomas, three developed distant metastases and the smallest one was $13.25 \mathrm{~mm}$, harboring EGFR mutation in exon 19.

Nakamura et al. (12) firstly studied the relationship between VDT and EGFR mutation by retrospectively reviewing 102 NSCLCs and found that the VDTs of patients with EGFR mutations (median 676 days) were longer than those without EGFR mutations (median 139 days). However, the present study demonstrated that the median VDT of EGFR wide type group, EGFR mutation in exon 19 and exon 21 groups were 207, 288 and 144 days, respectively. We found whether there was EGFR mutation and where the mutation occurred generally didn't affect doubling time of adenocarcinomas. Investigating further, the different outcomes may be related to different histology subtypes in studied groups. Doubling time of lung cancer has been reported to be different among different histology subtypes (17) and doubling time of squamous cell carcinomas (SCC) has been reported to be shorter than that of adenocarcinomas $(13,14,18,19)$. In Nakamura's study, patients with EGFR mutations comprised of 33 (94\%) adenocarcinomas, while the group without EGFR mutations included 22 SCC and 36 (54\%) adenocarcinomas. Therefore, it was possible to have a longer VDT in EGFRmutation positive group where adenocarcinoma was predominant. Similarly, Tanimoto et al. (20) found VDT of lung cancer in patients with chronic obstructive pulmonary disease (COPD) findings (median 998 days) tended to be shorter than that without COPD findings (median 2,226 days), while there was no SCC $(0 \%)$ in non-COPD group but $10(38 \%)$ in COPD group. In our study of all adenocarcinomas, however, significant difference wasn't observed between non-COPD group and COPD group $(\mathrm{P}=0.25)$. Therefore, it may be advisable to take histology subtypes into consideration when analyzing VDT among different groups.

One important aim of the current study was to throw 


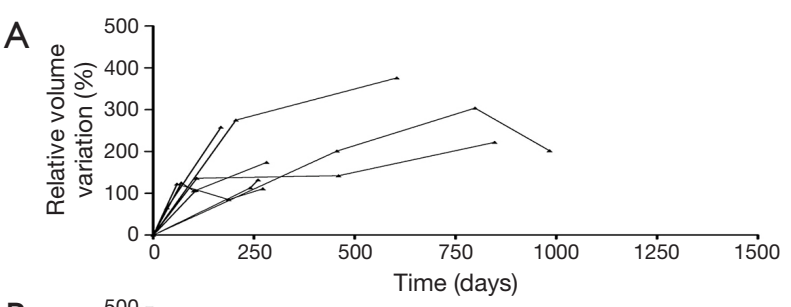

B
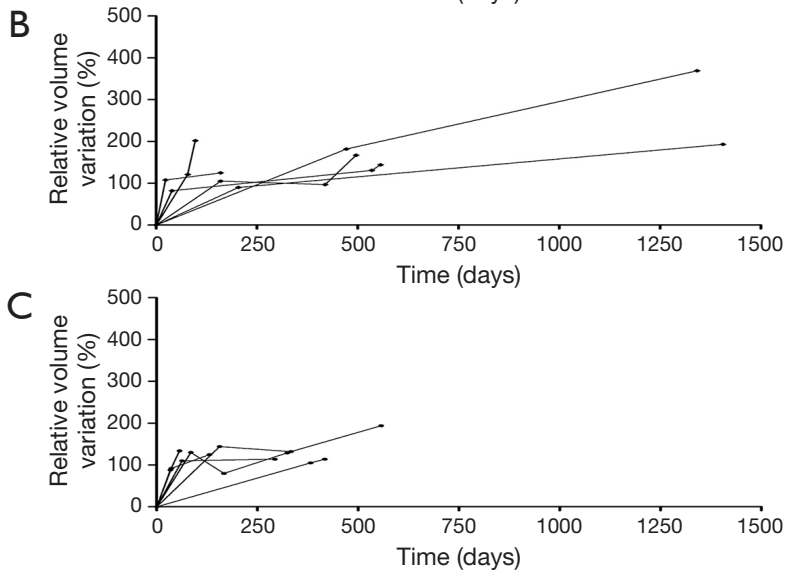

Figure 1 Growth curves of 20 adenocarcinomas with three or more follow-up scans. $\mathrm{Y}$ axis represents relative volume variation (\%), the ratio of subsequent follow-up volume to the initial volume. $\mathrm{X}$ axis represents time (days). (A) Adenocarcinomas of EGFR wide type ( $\mathrm{n}=8)$; (B) adenocarcinomas with EGFR mutation in exon $19(\mathrm{n}=6)$; (C) adenocarcinomas with EGFR mutation in exon $21(n=6)$. CT, computed tomography; EGFR, epidermal growth factor receptor.

more light on growth variability among EGFR wide type group, EGFR mutation in exon 19 and exon 21 groups using three-dimensional reconstruction software. As mentioned before, previous studies have already revealed that EGFR mutation status is closely related to the manifestation and prognosis of lung adenocarcinomas. Most recently, Liu et al. (2) retrospectively evaluated 385 surgically resected lung adenocarcinomas and found that 16 CT features were associated with EGFR mutation status. The most significant and independent prognostic factors of EGFRactivating mutation in their study were non-smokers, smaller tumor size, bubblelike lucency, homogenous enhancement or pleural retraction. Yano et al. (21) reported EGFR mutation was more frequently found in small peripheral adenocarcinomas with a GGO ratio of $50 \%$ or more, especially in females. Besides, Shi et al. (3) found sex, a small-maximum diameter, pleural retraction and the absence of fibrosis were associated with EGFR mutation in exon 19; non-smoker, spiculation, and subsolid density were more likely related to mutation in exon 21 . In our study, overall VDT of lung adenocarcinomas seems not affected by EGFR mutation status. To further identify growth difference among EGFR wide type group, EGFR mutation in exon 19 and exon 21 groups, subgroup analyses regarding to clinicopathoradiological parameters were performed. We noticed that, for males and patients without spiculation sign, VDT distributions were significantly different between EGFR mutation in exon 19 group and EGFR wide type group. The median VDT of exon 19 group was longer than that of wide type group and all adenocarcinomas with EGFR mutation in exon 19 showed a slowly growing tendency. This could be consistent with clinical manifestation mentioned above, such as female, non-smoker and small tumor size. Besides, slow growth could also contribute to superior prognosis of adenocarcinoma patients who harbor EGFR mutation in exon 19 (5). Actually, VDT varied among three groups at plenty of levels, though statistical differences weren't observed at most of levels. That could be related to small study population. For example, the VDT of adenocarcinomas presented as pGGO and mGGO was 716, 171, and 140 days for EGFR wide type group, EGFR mutation in exon 19 and exon 21 groups, respectively. It seemed that subsolid adenocarcinomas without EGFR mutation were less aggressive from these data. Shortly, it is expected that future studies with large study population will reveal valuable relationships between VDT and EGFR mutation status as clinicopathoradiological parameters are taken into consideration.

Negative VDTs weren't rare and reported in several previous studies $(10,14,17,19,22)$. Jennings et al. (10) reported 21 tumors that did not show growth and the relative volume decreased ranged from $1 \%$ to $81 \%$. Lindell et al. (19) examined 61 lung cancers and found four negative VDTs. They were all adenocarcinomas and became smaller according to both visual and computer assisted assessment. Recently, Mackintosh et al. (17) reported negative VDTs of three adenocarcinomas and one of those demonstrated actual tumor regression after histological examinations. In current study, 24 adenocarcinomas presented negative VDTs and relative volume decreased ranged from $1 \%$ to $53 \%$, most of which tended to stay stable instead of showing visible shrinkage. However, one patient harboring multiple mutations (exon 21, exon 20 and exon 18) did show a little bit shrinkage and its mass increased at the same time. The tumor was poorly differentiated and inflammatory reaction wasn't observed between two CT scans (Figure 4). Tumor regression, a complete or partial 
Table 3 Clinicopathoradiologic characters and VDT ${ }^{a}$ distribution among three groups ${ }^{\mathrm{b}}$

\begin{tabular}{|c|c|c|c|c|}
\hline Characteristics & Number & VDT wide type [days] & VDT mutation in exon 19 [days] & VDT mutation in exon 21 [days] \\
\hline Male & 33 & $164[87-2,506]$ & $273[91-3,749]$ & $351[109-4,148]^{\mathrm{e}}$ \\
\hline Female & 55 & $318[55-10,920]$ & $303[153-1,508]$ & $139[61-2,171]$ \\
\hline \multicolumn{5}{|l|}{ Smoking history } \\
\hline Smoker & 25 & $164[55-2,506]$ & $603[99-3,749]$ & $351[109-4,148]$ \\
\hline \multicolumn{5}{|l|}{ Clinical stage } \\
\hline Stage I/II [55] & 55 & $317[55-10,920]$ & $296[100-4,148]$ & $145[91-3,749]$ \\
\hline Stage III/IV & 33 & 200 [61-858] & $237[87-1,508]$ & $550[135-2,506]$ \\
\hline Others $^{c}$ & 22 & 356 [107-657] & 209 [99-318] & $144[92-2,506]$ \\
\hline \multicolumn{5}{|l|}{ Histological grade } \\
\hline Low & 14 & $2,171^{d}$ & 214 [109-318] & $133[92-2,506]$ \\
\hline Moderate & 21 & 238 [61-878] & 160 [99-220] & $135[122-1,369]$ \\
\hline High & 13 & $408[55-10,920]$ & $891[273-1,508]$ & $455[171-3,749]$ \\
\hline \multicolumn{5}{|l|}{ Tumor texture } \\
\hline pGGO or mGGO & 29 & $716[95-10,920]$ & $171[91-1,085]$ & $140[75-4,148]$ \\
\hline Solid & 59 & $172[55-2,506]$ & $303[99-3,749]$ & $157[61-2,171]$ \\
\hline Yes & 45 & 172 [55-10920] & $858[99-3,749]$ & $157[61-2,171]$ \\
\hline \multicolumn{5}{|l|}{ Air bronchogram } \\
\hline No & 62 & $161[55-10,920]$ & $288[91-3,749]$ & 157 [107-878] \\
\hline Yes & 26 & $290[87-1,236]$ & $506[99-1,085]$ & $134[61-4,148]$ \\
\hline \multicolumn{5}{|l|}{ Emphysema } \\
\hline No & 58 & $187[55-1,236]$ & $499[122-3,749]$ & $157[61-4,148]$ \\
\hline Yes & 30 & $290[92-10,920]$ & $213[91-1,085]$ & 140 [107-878] \\
\hline
\end{tabular}

${ }^{a}$, the median and range for VDT didn't include negative values $(n=24) ;{ }^{b}$, EGFR wide type group, EGFR mutation in exon 19 and 21 groups; calculated with the Kruskal-Wallis test; ${ }^{c}$, mainly acinar predominant; ${ }^{d}$, only one positive data available; ${ }^{e}$, significant difference $(\mathrm{P}<0.05)$. VDT, volume doubling time; pGGO, pure ground glass opacity; mGGO, mixed ground glass opacity. 

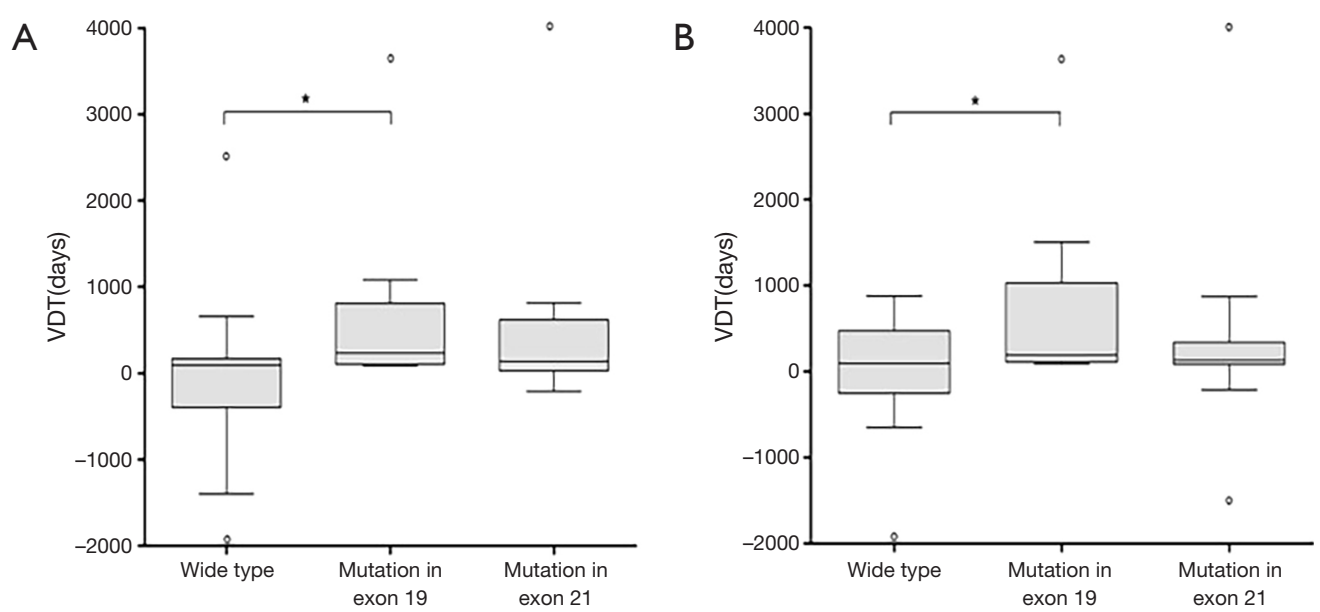

Figure 2 Box plot shows VDTs for EGFR wide type group, EGFR mutation in exon 19 group and exon 21 group. The upper and lower ends of the whiskers, the horizontal lines across each box, and circles represent upper and lower extremes, medians, and data outliers, respectively. *, $\mathrm{P}<0.05$. (A) VDTs for studied males ( $\mathrm{P}=0.03)$; (B) VDTs for studied patients without spiculation sign in chest computed tomography $(\mathrm{P}=0.04)$. VDTs, volume doubling times; EGFR, epidermal growth factor receptor.

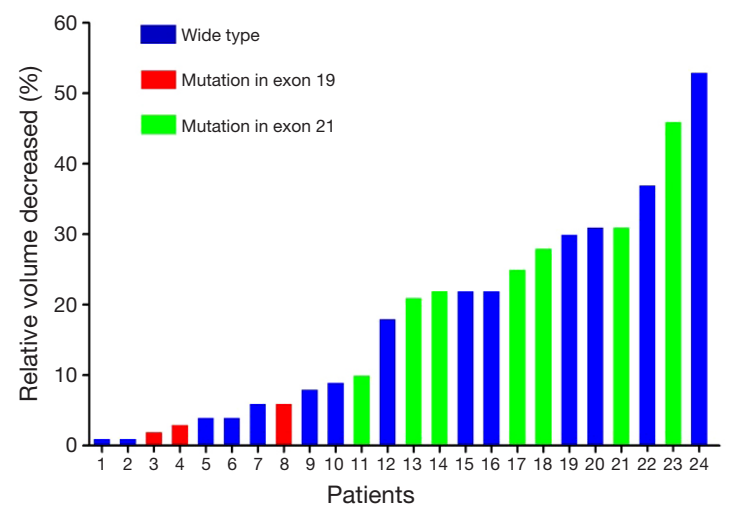

Figure 3 Relative volume decreased of studied 24 adenocarcinomas in 24 patients. $\mathrm{Y}$ axis represents relative volume decreased (\%), the ratio of decreased volume (the difference between the last and initial volume) to the initial volume. $\mathrm{X}$ axis represents 24 patients.

disappearance of malignant tumors without any treatment, is not rare and frequently reported in carcinoma of the urinary bladder, renal adenocarcinoma, embryonal and breast cancer, neuroblastoma, melanoma and sarcoma (22). There were also case reports of tumor regression in lung cancer $(23,24)$. The regression phenomena may be associated with bacterial, viral, fungal infection, which can stimulate the immune system of our body. For example, a previous study showed that patients with lung cancer and tuberculosis (TB) at the same time survived longer than those without TB (25). Besides, necrosis, implosion, angioneogenesis, reexpansion of adjacent lung region may also be associated with lung cancer regression (17).

The current study has two advantages. Firstly, all patients included were diagnosed with adenocarcinomas. That avoided statistical differences caused by histology subtypes instead of a real interesting factor. Besides, threedimensional volumetric software was applied to perform tumor volume measurements, which can be more accurate and reliable. However, there are also several limitations. Firstly and most importantly, it was a small number of study and some real differences may be neglected. Due to our restricted inclusion criterion, limited cases were selected and many factors of interest were out of our reach. Secondly, intraobserver agreement was not evaluated and volume change caused by manual nodule segmentation was unable to assess. Thirdly, chest CT scans in our study were taken by three different scanners, although images of each patient were paired according to the thickness of films. Whether the patient was scanned with or without contrast, it was always advisable to select CT images scanned by the same scanner under same protocols. Fourthly, 24 patients in our study underwent transbronchial lung biopsy between two CT scans, which may influence volume measurements, especially when the measurement was done shortly after the diagnostic biopsy. However, that didn't occur in our cases and may not significantly alter our results. Seven (29.2\%) of them had a negative VDT. Last but not the least, the scan interval for some patients wasn't long enough. It's better to 

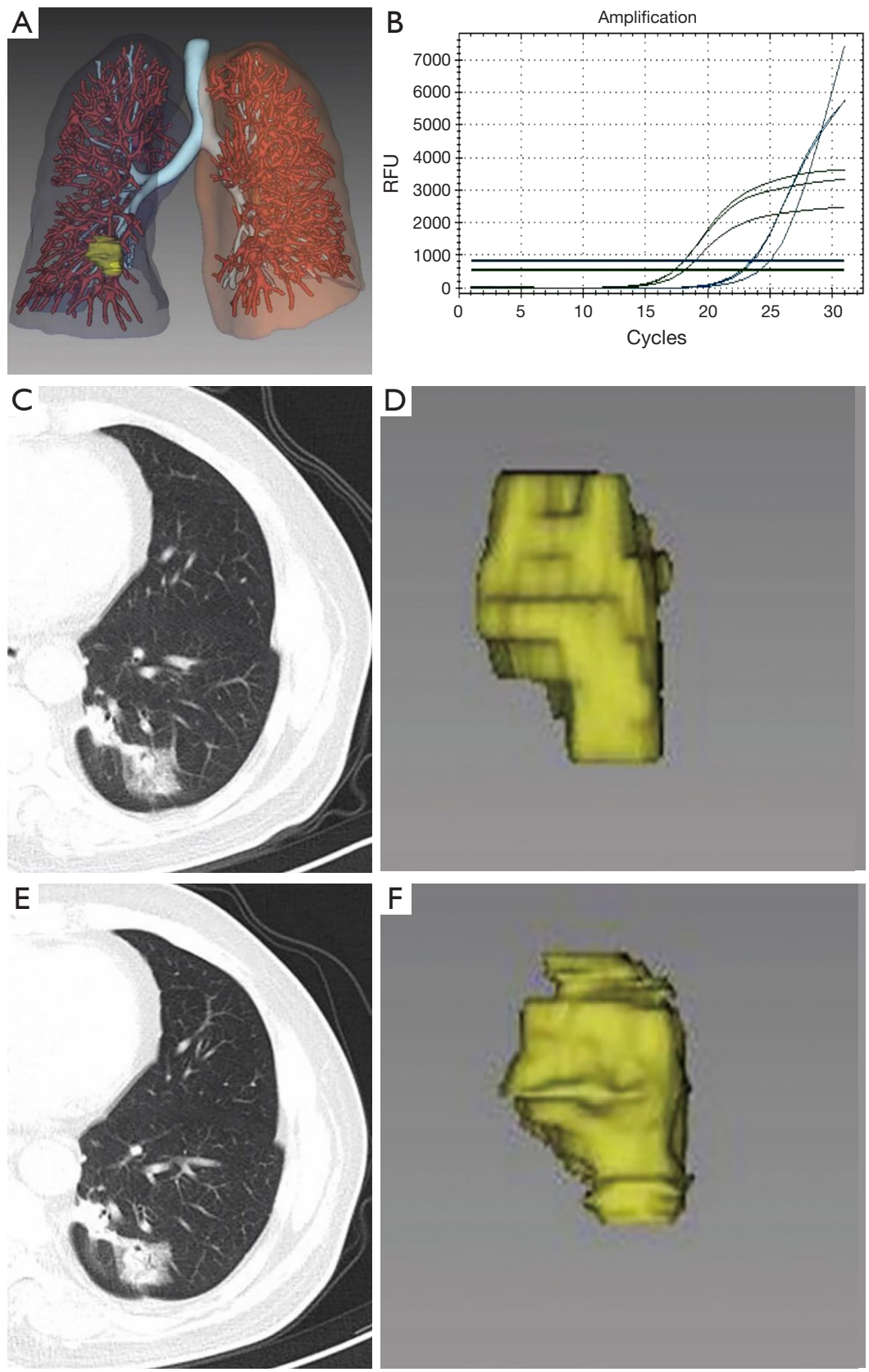

Figure 4 Adenocarcinoma in a 69-year-old woman with EGFR mutation in exon 21, exon 20 and exon 18. The tumor decreased in size but its mass increased, VDT $=-210$ days. (A) Three-dimension reconstruction of the tumor in the left lower lobe, posteriorly (IQQA-Chest, EDDA Technology, Princeton Junction, NJ, USA); (B) detection of EGFR mutation status (Cycleave real-time quantitative PCR technique); (C) initial CT scan of the tumor; (D) three-dimension reconstruction of the tumor in initial CT scan, V=13,966 mm ${ }^{3}, \mathrm{~m}=2.12 \mathrm{~g}$; (E) second CT scan of the tumor after 86 days; (F) three-dimension reconstruction of the tumor in the second CT scan, V=10,521 mm ${ }^{3}, \mathrm{~m}=10.01 \mathrm{~g}$, $\Delta \mathrm{V}=-3445 \mathrm{~mm}^{3}, \Delta \mathrm{M}=7.89 \mathrm{~g}$. EGFR, epidermal growth factor receptor; VDT, volume doubling time. 
have scan interval longer than 3 months.

\section{Conclusions}

Our study found the median VDT for lung adenocarcinomas was 214 days, except for negative VDTs. Whether there was EGFR mutation and where the mutation occurred generally didn't affect the overall doubling time. However, the median VDT of adenocarcinomas with EGFR mutation in exon 19 was longer than that of wide type among males or patients without spiculation sign. Due to the small sample size, studies with large population are warranted to reveal valuable relationships between VDT and EGFR mutation status as clinicopathoradiological parameters are taken into consideration.

\section{Acknowledgements}

Funding: This work was supported by Achievements Transformation Program of Sichuan Province (2016CZYD0001).

\section{Footnote}

Conflicts of Interest: The authors have no conflicts of interest to declare.

Ethical Statement: The study was approved by the Medical Ethical Committee of West China Hospital, Sichuan University (IRB: 2016/151).

\section{References}

1. Nicholson RI, Gee JM, Harper ME. EGFR and cancer prognosis. Eur J Cancer 2001;37 Suppl 4:S9-15.

2. Liu Y, Kim J, Qu F, et al. CT Features Associated with Epidermal Growth Factor Receptor Mutation Status in Patients with Lung Adenocarcinoma. Radiology 2016;280:271-80.

3. Shi Z, Zheng X, Shi R, et al. Radiological and Clinical Features associated with Epidermal Growth Factor Receptor Mutation Status of Exon 19 and 21 in Lung Adenocarcinoma. Sci Rep 2017;7:364.

4. Ke EE, Zhou Q, Zhang QY, et al. A Higher Proportion of the EGFR T790M Mutation May Contribute to the Better Survival of Patients with Exon 19 Deletions Compared with Those with L858R. J Thorac Oncol 2017;12:1368-75.

5. Liu Y, Ren Z, Wang J, et al. Epidermal growth factor receptor-tyrosine kinase inhibitor therapy is especially beneficial to patients with exon 19 deletion compared with exon $21 \mathrm{~L} 858 \mathrm{R}$ mutation in non-small-cell lung cancer: Systematic review and meta analysis. Thorac Cancer 2016;7:406-14.

6. Choi YW, Jeon SY, Jeong GS, et al. EGFR Exon 19 Deletion is Associated With Favorable Overall Survival After First-line Gefitinib Therapy in Advanced Non-Small Cell Lung Cancer Patients. Am J Clin Oncol 2016. [Epub ahead of print].

7. Zhang Y, Chen G, Chen X, et al. The comparison of EGFR-TKI failure modes and subsequent management between exon 19 deletion and exon 21 L858R mutation in advanced non-small-cell lung cancer. J Cancer 2017;8:1865-71.

8. Tuttle RM, Fagin JA, Minkowitz G, et al. Natural History and Tumor Volume Kinetics of Papillary Thyroid Cancers During Active Surveillance. JAMA Otolaryngol Head Neck Surg 2017;143:1015-20.

9. de Laat JM, Pieterman CR, van den Broek MF, et al. Natural course and survival of neuroendocrine tumors of thymus and lung in MEN1 patients. J Clin Endocrinol Metab 2014;99:3325-33.

10. Jennings SG, Winer-Muram HT, Tann M, et al. Distribution of stage I lung cancer growth rates determined with serial volumetric CT measurements. Radiology 2006;241:554-63.

11. Song YS, Park CM, Park SJ, et al. Volume and mass doubling times of persistent pulmonary subsolid nodules detected in patients without known malignancy. Radiology 2014;273:276-84.

12. Nakamura R, Inage $Y$, Tobita R, et al. Epidermal growth factor receptor mutations: effect on volume doubling time of non-small-cell lung cancer patients. J Thorac Oncol 2014;9:1340-4.

13. Veronesi G, Maisonneuve P, Bellomi M, et al. Estimating overdiagnosis in low-dose computed tomography screening for lung cancer: a cohort study. Ann Intern Med 2012;157:776-84.

14. Honda O, Johkoh T, Sekiguchi J, et al. Doubling time of lung cancer determined using three-dimensional volumetric software: comparison of squamous cell carcinoma and adenocarcinoma. Lung Cancer 2009;66:211-7.

15. Geddes DM. The natural history of lung cancer: a review based on rates of tumour growth. Br J Dis Chest 1979;73:1-17.

16. Usuda K, Saito Y, Sagawa M, et al. Tumor doubling time and prognostic assessment of patients with primary lung 
cancer. Cancer 1994;74:2239-44.

17. Mackintosh JA, Marshall HM, Yang IA, et al. A retrospective study of volume doubling time in surgically resected non-small cell lung cancer. Respirology 2014;19:755-62.

18. Hasegawa M, Sone S, Takashima S, et al. Growth rate of small lung cancers detected on mass CT screening. Br J Radiol 2000;73:1252-9.

19. Lindell RM, Hartman TE, Swensen SJ, et al. Five-year lung cancer screening experience: CT appearance, growth rate, location, and histologic features of 61 lung cancers. Radiology 2007;242:555-62.

20. Tanimoto D, Ito K, Tamada T, et al. Serial 3-dimensional volumetric computed tomography evaluation of lung cancer growth rate in patients with chronic obstructive pulmonary disease findings. J Comput Assist Tomogr 2012;36:181-6.

21. Yano M, Sasaki H, Kobayashi Y, et al. Epidermal growth

Cite this article as: Zhang R, Chen B, Zhou Y, Zhou P, Jin J, Zhao L, Li W. Volume doubling time of lung adenocarcinomas considering epidermal growth factor receptor mutation status of exon 19 and 21: three-dimensional volumetric evaluation. J Thorac Dis 2017;9(11):4387-4397. doi: 10.21037/ jtd.2017.10.58 factor receptor gene mutation and computed tomographic findings in peripheral pulmonary adenocarcinoma. J Thorac Oncol 2006;1:413-6.

22. Quint LE, Cheng J, Schipper M, et al. Lung lesion doubling times: values and variability based on method of volume determination. Clin Radiol 2008;63:41-8.

23. Horino T, Takao T, Yamamoto M, et al. Spontaneous remission of small cell lung cancer: a case report and review in the literature. Lung Cancer 2006;53:249-52.

24. Hwang ED, Kim YJ, Leem AY, et al. Spontaneous regression of non-small cell lung cancer in a patient with idiopathic pulmonary fibrosis: a case report. Tuberc Respir Dis (Seoul) 2013;75:214-7.

25. Kappauf H, Gallmeier WM, Wunsch PH, et al. Complete spontaneous remission in a patient with metastatic nonsmall-cell lung cancer. Case report, review of the literature, and discussion of possible biological pathways involved. Ann Oncol 1997;8:1031-9. 